\title{
A sparse coding model of V1 produces surround suppression effects in response to natural scenes
}

\author{
Allison Del Giorno ${ }^{1 *}$, Mengchen Zhu², Christopher J Rozell ${ }^{1}$ \\ From Twenty Second Annual Computational Neuroscience Meeting: CNS*2013 \\ Paris, France. 13-18 July 2013
}

While many neural coding models have been proposed for V1, it remains an open question as to which model best describes the diversity of observed response properties. For instance, the canonical linear-nonlinear model (LN) partially explains some fundamental mechanistic and phenomenological properties of $\mathrm{V} 1$, but is unable to explain many nonlinear response properties that are likely associated with the keys to efficient and robust human vision.

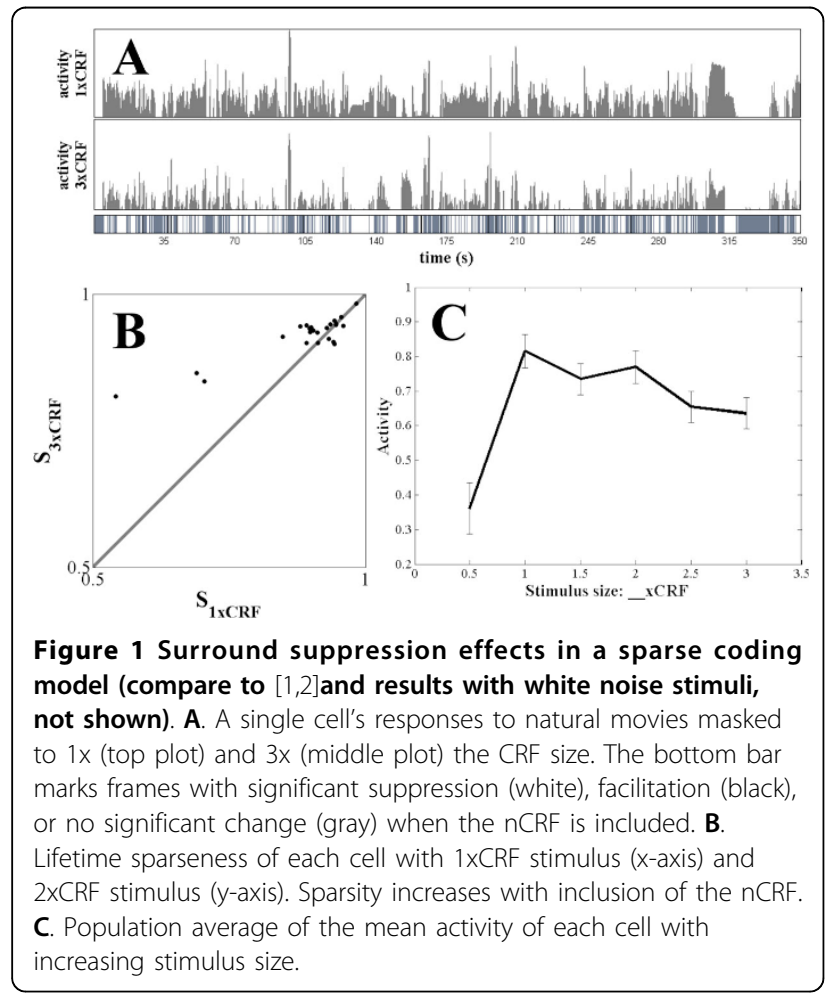

\footnotetext{
* Correspondence: allie.delgiorno@gatech.edu

${ }^{1}$ Electrical and Computer Engineering, Georgia Institute of Technology, Atlanta, GA 30332, USA

Full list of author information is available at the end of the article
}

For example, surround suppression is one such nonlinear response property in which visual stimuli extending beyond the classical receptive field (CRF) selectively diminish neural responses. This property has been studied through electrophysiology experiments with synthetic stimuli (e.g., gratings). Surprisingly, high level sparse coding models implemented in a biologically plausible dynamical system have been shown to produce surround suppression effects that match individual and population observed responses [3]. More recently, surround suppression has been investigated experimentally using natural stimuli, and these experiments have shown an increase in the sparsity of measured responses [1,2]. Despite these findings, it remains unclear whether a functional sparse coding model is sufficient to produce the types of surround suppression observed with natural stimuli. In this abstract, we demonstrate that the surround suppression effects recently observed with natural stimuli are also emergent properties of a sparse coding model (Figure 1).

\section{Author details}

${ }^{1}$ Electrical and Computer Engineering, Georgia Institute of Technology, Atlanta, GA 30332, USA. Biomedical Engineering, Georgia Institute of Technology, Atlanta, GA 30332, USA.

Published: 8 July 2013

\section{References}

1. Haider B, Krause MR, Duque A, Yu Y, Touryan J, Mazer J, McCormick D: Synaptic and network mechanisms of sparse and reliable visual cortical activity during nonclassical receptive field stimulation. Neuron 2010, 65:107-21.

2. Vinje $W E$, Gallant $J$ : Sparse coding and decorrelation in primary visual cortex during natural vision. Science (New York, NY) 2000, 287:1273-6.

3. Zhu M, Rozell C: Biologically realistic excitatory and inhibitory cell properties emerge from a sparse coding network [abstract, CNS 2012]. BMC Neuroscience 2012, 13(Suppl 1):P55.

doi:10.1186/1471-2202-14-S1-P335

Cite this article as: Giorno et al:: A sparse coding model of V1 produces surround suppression effects in response to natural scenes. $B M C$ Neuroscience 2013 14(Suppl 1):P335.

\section{() Biomed Central}

(c) 2013 Giorno et al; licensee BioMed Central Ltd. This is an Open Access article distributed under the terms of the Creative Commons Attribution License (http://creativecommons.org/licenses/by/2.0), which permits unrestricted use, distribution, and reproduction in any medium, provided the original work is properly cited. 